\title{
Multistability in networks of Hindmarsh-Rose neurons
}

\author{
R. Erichsen, Jr. ${ }^{*}$ and L. G. Brunnet ${ }^{\dagger}$ \\ Instituto de Física, Universidade Federal do Rio Grande do Sul, Caixa Postal 15051, 91501-970 Porto Alegre, RS, Brasil
}

(Received 2 May 2008; revised manuscript received 30 September 2008; published 18 December 2008)

\begin{abstract}
We investigate the dynamical states of a two-dimensional network of Hindmarsh-Rose spiking neurons, in the vicinity of the current threshold where the single neuron becomes active. Each neuron is electrically coupled with neurons in its close neighborhood. The existence of multistable synchronization states is established and discussed. We also show that, provided adequate initial conditions, the collective behavior is able to keep the network in activity, even for current values far below the activity threshold of the single neuron. A phase diagram of the different network states is presented for a large interval of the coupling-current parameter space.
\end{abstract}

DOI: 10.1103/PhysRevE.78.061917

\section{INTRODUCTION}

The whole brain is a complex system composed of subunits specialized to perform different but integrated tasks. Apart from this complexity, it is common sense among neuroscientists the assumption that all computational ability of a living brain emerges from the collective behavior of a large set of spiking neurons on each of its subunits.

A realistic model of a biological neuron was proposed by Hindmarsh and Rose (HR) [1]. The isolated HR neuron has the ability to reproduce distinct biological neuronal activities such as quiescence, regular or chaotic spikes, and regular or chaotic spike trains with low computational cost. A simple system composed of two interacting HR neurons shows a rich dynamical activity [2-4]. A two-dimensional (2D) network of HR neurons with nearest-neighbors electrical coupling is an example of an excitable medium showing bistability $[5,6]$. This means that a globally synchronized stationary state may be reached evolving from random initial conditions; but also, a partially synchronized stationary state, where spirals and traveling waves are formed, can be attained following an appropriate initial annealing procedure [5]. Whether the coding in the cortex uses either spike trains or individual spikes, either partial or global synchronization, is presently a matter of research [7]. Recently, it has been argued that partial synchronization could explain the large dynamic range of sensory cortex [8].

In order to qualify the HR model as a suitable model for information coding, it is worth studying the interplay among quiescence, global synchronization, and partial synchronization in a model network. Unfortunately, to our knowledge, no analytical treatment with this purpose has been developed yet. In this paper, results of a numerical investigation of the different synchronization states of the 2D HR model, in the vicinity of the activity threshold, are reported. The main emphasis is devoted to the elaboration of a phase diagram showing the dominant dynamical attractors, which are identified by simple, but powerful, measuring tools. The paper is organized as follows: in the next section the HR model is recalled and its assembling in the network is presented. In

\footnotetext{
*rubem@if.ufrgs.br

†leon@if.ufrgs.br
}

PACS number(s): 87.19.lj, 87.19.lg

Sec. III we show the distinction between global and partial synchronization; macroscopic measures aiming to identify these network states are introduced, and the main results are presented and discussed. Some concluding remarks can be found at the end of the paper.

\section{MODEL}

We deal in this paper with a simple model of the brain cortex composed of a 2D square network $\mathcal{N}$ of $N=L \times L \mathrm{HR}$ neurons. The time evolution of the neuron labeled $i \in \mathcal{N}$ is described by the set of equations

$$
\begin{gathered}
\frac{d x_{i}}{d t}=y_{i}+3 x_{i}^{2}-x_{i}^{3}-z_{i}+I+\frac{\epsilon}{|\mathcal{V}|} \sum_{j \in \mathcal{V}}\left(x_{j}-x_{i}\right), \\
\frac{d y_{i}}{d t}=1-5 x_{i}^{2}-y_{i}, \\
\frac{d z_{i}}{d t}=-r z_{i}+r S\left(x_{i}+1.618\right) .
\end{gathered}
$$

Variable $x_{i}$ represents the membrane potential. Variables $y_{i}$ and $z_{i}$ represent, respectively, "fast" and "slow" ion currents in the neuron dynamics. Constants $r$ and $S$ are model parameters adjusted in order to approximate the biological behavior. The parameter $I$ accounts for an external exciting current following in vitro measure protocol. The electric coupling with $|\mathcal{V}|$ neurons belonging to the neighborhood $\mathcal{V}$ is introduced with the addition of an ohmic term to the first equation for each neuron. The parameter $\epsilon$ plays the role of a uniform conductivity. We define the neighborhood of neuron $i$ as $\{j$ $\left.\in \mathcal{N}:\left|\mathbf{r}_{j}-\mathbf{r}_{i}\right| \leqslant R\right\}$, where $R$ is the interaction range. Periodic boundary conditions are imposed. Through all this work, we assume the literature values $r=0.0021$ and $S=4$ (see Ref. [5]). The model equations were integrated using a variable step fourth-order Runge-Kutta algorithm.

In its first version, the isolated HR neuron model was derived from the FitzHugh-Nagumo model $[9,10]$ adapted to account for the observed "tail current reversal" in voltage clamp experiments [11]. This system, composed of two firstorder ordinary differential equations, presents as solutions either a fixed point (subthreshold stable resting state) or a 

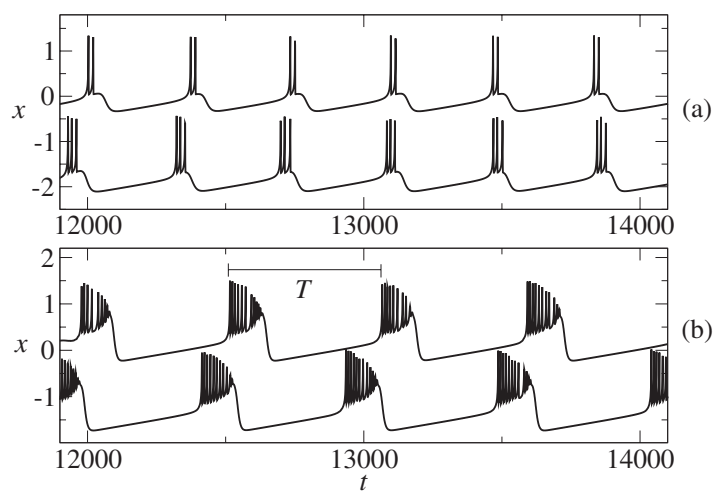

FIG. 1. Local activities of networked neurons, from square networks with $L=32$ and $R=2$. (a) Single-neuron-type activity, $\epsilon$ $=0.02, I=1.37$ (upper) and $I=1.375$ (lower). (b) Collective-type activity, $\epsilon=0.18, I=1.37$ (upper) and $I=1.375$ (lower). $T$ is the train period.

limit cycle (stable firing), depending on the external current applied. In the search for a model exhibiting triggered firing, an adaptation variable [the last of Eqs. (1)] with a long time constant was added changing the applied current $I$ to an effective one, $I-z$. In this manner, above some minimal current, the system shows a spiking behavior followed by a long resting state [1].

Stemming from this construction, the single HR neuron shows a quiescent fixed-point solution for low currents. From the eigenvalues of the Jacobian of Eqs. (1) evaluated at such low currents, we have found that this solution becomes unstable for $I^{*} \gtrsim 1.3616$. In fact, at this fixed point, one of the eigenvalues is negative $\left(\lambda_{1} \sim-14.2030\right)$, indicating strong contraction in one direction, while the two other, $\lambda_{2,3}$, are complex conjugate with null real part, suggesting a twodimensional bifurcation. In the interval of currents just below the critical value, $1.3408 \leqq I \leqq I^{*}$, the real part of $\lambda_{2,3}$ is always negative, but the basin of attraction to this fixed point is finite and shrinks to a point at $I^{*}$. This scenario is akin to a subcritical Hopf bifurcation where two solutions coexist for adequate initial conditions [12]. As we show below, this feature is important to understand the network activity close to this edge.

\section{RESULTS AND DISCUSSION}

When $N$ neurons are coupled, a $3 N$-dimensional phase space should be considered. Nevertheless, for small conductivity $\epsilon \lesssim 0.15$ the wired neuron keeps some of the characteristics of the isolated neuron. Indeed, it follows a spike bursting activity with a small and well-defined $I$ and $\epsilon$-dependent number of spikes per burst. In consequence, we call the local attractor, in this case, as being of the single-neuron type. Examples for two near conditions are shown in Fig. 1(a). The upper (lower) curve corresponds to $I=1.37(I=1.375)$ and $\epsilon=0.02$, although in the vicinity in the space of parameters, there is a quantitative difference between the two curves. As $\epsilon$ increases, the number of spikes per burst saturates, and for $\epsilon \gtrsim 0.15$ the activity is less sensitive to the network parameters. Two examples are shown in Fig. 1(b). The upper (lower) curve corresponds to $I=1.370 \quad(I=1.380)$ and $\epsilon$ $=0.18$. Since this kind of activity can be seen only in a connected neuron at large coupling regime, $(\epsilon \gtrsim 0.15)$, we call the corresponding local attractor as being of the collective type.

Besides distinguishing among single-neuron- and collective-type attractors, we also observe two different kinds of network synchronization states: global and partial. In the former, all neurons are found in only one of the activity states: spiking or quiescence. In the latter, neurons are found in both activity states at the same time throughout the network.

In order to quantitatively describe the network states and clarify the meaning of single-neuron- and collective-type attractors, as well as global and partial synchronization, we define two order parameters: (i) the membrane potential variance,

$$
m=\left\langle x_{i}^{2}-\left\langle x_{i}\right\rangle_{t, \mathcal{N}}^{2}\right\rangle_{t, \mathcal{N}},
$$

and (ii) the membrane potential covariance,

$$
q=\left\langle x_{i} x_{j}-\left\langle x_{i}\right\rangle_{t, \mathcal{N}}^{2}\right\rangle_{t, \mathcal{N}}
$$

with \langle\rangle$_{t, \mathcal{N}}$ meaning time and network averages. The membrane potential variance $m$ is a measure of the average network activity, and the membrane potential covariance $q$ is a measure of the average global network correlation.

The above order parameters allow us to clearly identify the just mentioned dynamic network states and attractors: (i) when $m=0$, all sites have a constant and uniform membrane potential. In that case, $q$ also has null value and the system is in the quiescent $(Q)$ state. (ii) If the lattice globally oscillates in time with a periodic instantaneous lattice averaged phase, $\langle\phi(t)\rangle_{\mathcal{N}} \simeq\langle\phi(t+T)\rangle_{\mathcal{N}}[6,13]$, where $T$ is the average bursting period (see Fig. 1), then $m \neq 0$ and $0<q \lessgtr m$. The system keeps spatial correlation, indicating a globally $(G)$ coherent state. (iii) When global spatial correlation is lost, only local correlations of a partially correlated $(P)$ state due to the local coupling are expected. That is, $m \neq 0$ and $q \simeq 0$. (iv) Singleneuron- and collective-type attractors can be identified directly by the network activity $m$ the former showing lower activity than the latter. See the discussion below. Finally, (v) if all sites strictly synchronize $(S)$ either in a periodic or chaotic dynamics, then at any given time $x_{i}=x_{j}$ and Eqs. (2) and (3) would yield the same result, implying $m=q \neq 0$. It is worth remarking that the last case was not observed in the region of parameter space explored in this work.

The curves for the order parameters $m$ and $q$ in the function of $\epsilon$ for fixed $I=1.37$ are shown in Fig. 2. This figure results from simulations over periodic contour squared networks with $L=32$ and $R=2$. Here and in most of the paper, each curve was averaged over 10 runs, starting from random initial conditions. A transient of 200000 time units (t.u.) was discarded and time average was taken over a further 20000 time units. The steplike increase in the activity level $m, \epsilon$ $\approx 0.023$, indicates that the number of spikes in each burst changes abruptly from 2 to 3 through the whole network. The nonmonotonic behavior in $q$ indicates that the network loses global correlation when the number of spikes is near to a change. The next change, from three to four spikes, shows 


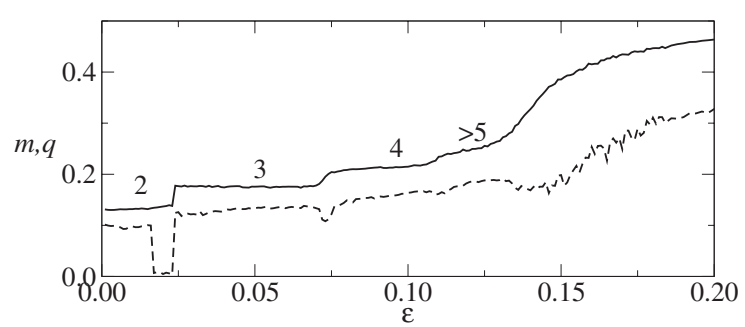

FIG. 2. Order parameters $m$ (solid line) and $q$ (dashed line) in a function of $\epsilon$ for $I=1.37$, for a square network with $L=32$ and $R$ $=2$. Numerals indicate the number of spikes per burst in each activity level. For more details concerning simulation conditions, see the text.

a finite slope in $m$. This suggests that there is a small region where these two attractors coexist. At such coupling values a small reduction in the correlation is observed in the $q$ curve. The change from four to five spikes is still less pronounced. After five spikes the activity level increases without steps. Pure five spikes is not observed, but each neuron bursts with five or more spikes. This is the upper limit where the singleneuron-type attractor can be found. After a soft transition in the range $0.13 \leqq \epsilon \lessgtr 0.16$, where the whole network activity shows a fast increase with several burst lengths coexisting, the network approaches a regime where the overall average activity increases in a moderate rate, with 13 or more spikes per burst. This is the locus of the collective-type attractor. To resume, it is possible to identify single-neuron- and collective-type attractors based on the activity level. In the first case we have $m \approx 0.2$, while in the second case we have $m \gtrsim 0.4$.

The curves for the order parameters as functions of $I$ for a low $\epsilon=0.04$ and a high $\epsilon=0.18$ are shown in Fig. 3. As in Fig. 2, the results refer to simulations over periodic contour square networks with $L=32$ and $R=2$. Simulations with a larger network $(L=64)$ and a larger interaction range $(R$ $=4)$ were also carried out, without significant changes. The curve for $\epsilon=0.04$, where a single-neuron dynamics dominates, is shown in Fig. 3(a). As the current $I$ increases, the network passes from quiescence with $m=q \simeq 0$ to global synchronization $m \gtrsim q>0$. As in Fig. 2, in the interval where

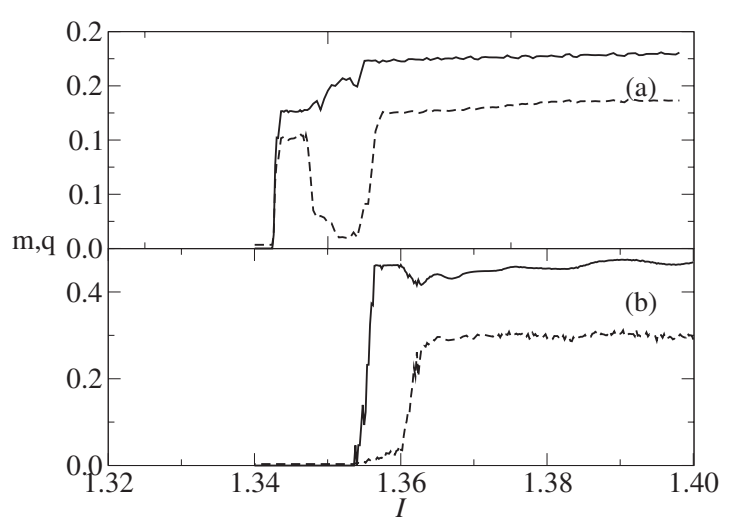

FIG. 3. Variance $m$ (solid lines) and covariance $q$ (dashed lines) as a function of the current $I$ for fixed $\epsilon=0.04$ (a) and $\epsilon=0.18$ (b), resulting from simulations of square networks with $L=32$ and $R$ $=2$.

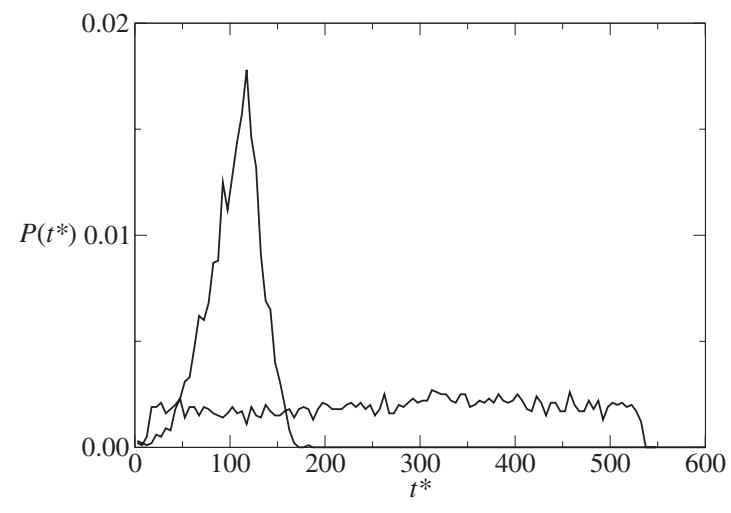

FIG. 4. Phase distribution for a square network with $L=128$, $R=2, I=1.37$, and $\epsilon=0.2$. Peaked (uniform) distribution results from $G(P)$ states. The time $t^{*}$ is the time elapsed since a reference time $t_{0}$.

$m>0$ and $q \simeq 0$ the global synchronization is lost. This happens when the local dynamics changes from two to three spikes per burst. The structure of the curves reveals the relevance of local neuron dynamics in this $\epsilon$ regime.

Figure 3(b), for $\epsilon=0.18$, gives an example of the general behavior in the large- $\epsilon$ regime, where the collective dynamics dominates. As $I$ increases there is a first transition, from quiescence to partial synchronization and then, at a higher $I$, there is a second transition, from partial to global synchronization. In the present case these two transitions are at $I$ $\simeq 1.355$ and $I \simeq 1.361$. Excepting small fluctuations in $m$, these curves do not reveal any important structure into each phase, a behavior at odds with the low- $\epsilon$ regime.

\section{A. Bistability}

As noticed in Refs. [5,6] for $I=3.28$, where the uncoupled neuron is chaotic, the network shows bistability between a global synchronization state, where the spike trains are strongly globally correlated and a partial synchronization state, where spike trains are only locally correlated and a space-time pattern formation occurs. We refer to those papers for detailed descriptions of these states. This section aims to show that bistability is already present in the low current regime, where the uncoupled neuron is periodic.

In addition to $m$ and $q$, the distribution of spike train phases $P\left(t^{*}\right)$ offers further characterization of global and partial synchronization. It is defined as follows: if an arbitrary reference time $t_{0}$ is taken, and the difference $t^{*}=t-t_{0}$, for the time when a given neuron starts a spike burst is recorded, then a phase $\phi=2 \pi t^{*} / T$ is attributed to that neuron. Normally, every neuron starts a burst at least once during a complete time interval equal to $T$. A complete set $\left\{t_{i}^{*}\right\}$ for $1 \leqslant i$ $\leqslant N$ can be obtained during a time interval $T$, after a given transient time. This allows for the construction of $P\left(t^{*}\right)$. The two resulting distributions found for $I=1.37$ and $\epsilon=0.2$ are shown in Fig. 4. There, peaked and uniform distributions correspond, respectively, to global and partial synchronizations. For these values of $I$ and $\epsilon$, when starting from random initial conditions, the system converges to global synchronization. Partial synchronization can be attained through a 


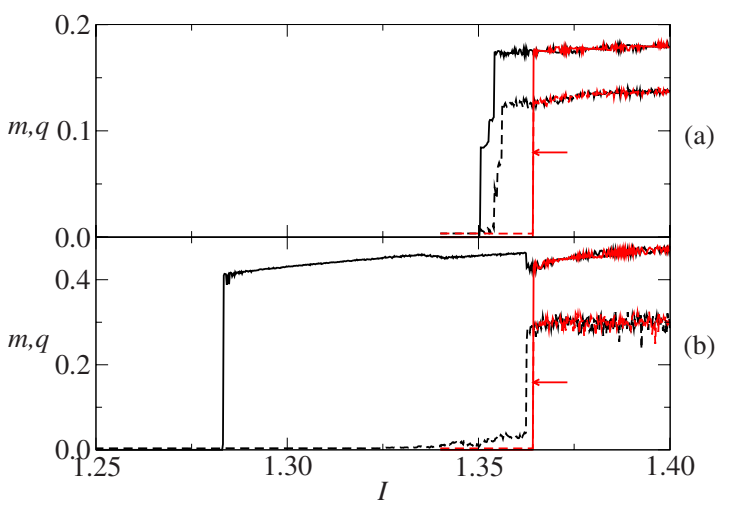

FIG. 5. (Color online) Variance $m$ (solid lines) and covariance $q$ (dashed lines) as a function of the current $I$ for fixed $\epsilon=0.04$ (a) and $\epsilon=0.18$ (b). Increasing $I$ is shown in gray (red) curves, with the transition from quiescence to global synchronization signaled by an arrow; decreasing $I$ is shown in black. The rate of change in $I$ is $1 \times 10^{-8}$ (t.u. $)^{-1}$. The network is the same as in Fig. 3 .

suited procedure, to be explained later in this work. From this figure, it is clear that in the partial synchronization state the neurons are uniformly out of phase, i.e., there is no phase clustering.

As a consequence of the bistability, the system reveals hysteresis, as can be appreciated in Fig. 5. There, curves of $m$ and $q$ for slowly increasing and decreasing $I$ at a rate of 1 $\times 10^{-8}$ (t.u. $)^{-1}$ and fixed $\epsilon$ are shown. The network is the same as in Fig. 3, but in Fig. 5 the dynamic state is preserved while $I$ changes. As in the previous section both low, $\epsilon$ $=0.04$ and high, $\epsilon=0.18$ regimes were investigated. Hysteresis is well characterized by different paths corresponding to increasing and decreasing $I$.

There are interesting results that come out from Fig. 5(b), where $\epsilon=0.18$. For increasing $I$, starting into the quiescent state, global synchronization is attained at $I \simeq 1.364$, as in Fig. 5(a). This value is very close to $I=1.362$, when quiescence becomes unstable in the single neuron. The coupling is proportional to the difference of cell potentials, and all cells have the same potential at quiescence. In consequence, the stability of the quiescent state should not depend on the $\epsilon$ value, and this seems to be indeed the case. Starting at $I$ $=1.4$ with random initial conditions the system reaches global synchronization. For decreasing $I$, partial synchronization is attained at $I \simeq 1.362$. We found that this is the case whenever $\epsilon \gtrsim 0.15$. Finally, quiescence is attained only at $I$ $\simeq 1.283$. It is important to remark that this value is significantly smaller than the overall lower limit of the activity state for the single neuron, $I \simeq 1.341$. As it will be shown later, the low limit of activity for decreasing $I$ decreases further for larger $\epsilon$. This is an important property of the collective-type attractor, and means that the collective behavior is able to keep the network active at $I$ levels far below that required by the uncoupled neuron.

The largest Lyapunov exponent (LLE) was numerically calculated following the method proposed in $[14,15]$. The results, shown in Fig. 6, correspond to the situation where the dynamical network state is preserved while $I$ slowly decreases. The parameters are the same as in Fig. 5. From this sample, we may infer that the active state has a chaotic evo-

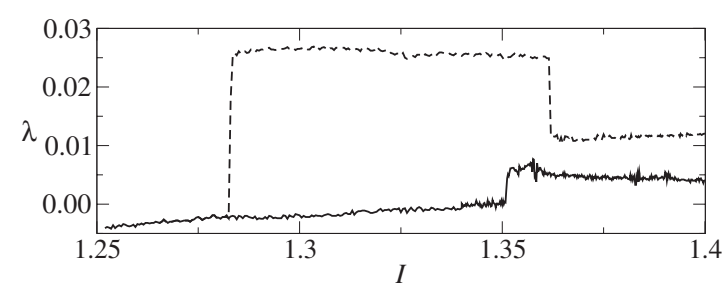

FIG. 6. Largest Lyapunov exponent $\lambda$ as a function of the current $I$ for $\epsilon=0.04$ (solid line) and $\epsilon=0.18$ (dashed line). The network is the same as in Figs. 3 and 5.

lution either in the low $\epsilon$, single-neuron-type attractor or in the high $\epsilon$, collective-type attractor. There are some important aspects to note in this figure. The LLE is positive, provided the network is in an active state. The LLE corresponding to partial synchronization is larger than that corresponding to global synchronization. This is what one could expect, since partial synchronization allows for spacetime pattern formation.

\section{B. Phase diagram}

The results presented so far for two particular values of the coupling $\epsilon$ is extended to a finite interval in the $\epsilon-I$ plane shown in Fig. 7. There, labels $G n$ and $P n$ identify, respectively, regions where single-neuron-type dynamics with $n$ spikes per train with global or partial synchronization can be found when starting from random initial conditions. Region $P 2$ appears in Fig. 2 as the interval where $q \approx 0$, and the transition from $G 3$ to $G 4$ appears, in that figure, as the depletion in $q$ and the step in $m$ near $\epsilon=0.071$. Label $G 4+$ identifies the region where there are spike trains with four and more spikes. For an increasing $\epsilon$, in the upper part of the diagram, we pass to the $G+P$ region. This transition is signaled in Fig. 2 as the smooth step in $m$ and the depletion in $q$ in the vicinity of $\epsilon \simeq 0.144$. It is determined by the appearance of the collective-type attractor. In general, the "+" signal means coexistence of phases. In $G+P$ global and partial synchronization coexist, but with random initial conditions the network reaches the global synchronization state $G$. In $Q$,

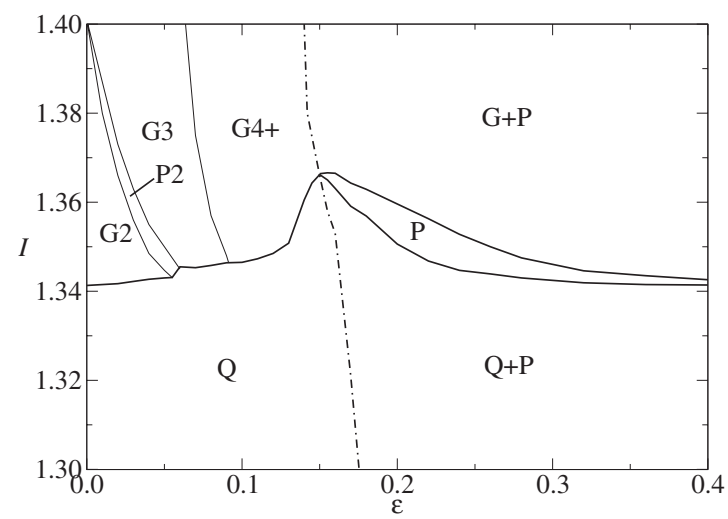

FIG. 7. $I$ vs $\epsilon$ diagram of a square network with $L=32$ and $R$ $=2$. Global synchronization, partial synchronization, and quiescence are identified, respectively, with the labels $G, P$, and $Q$. $G n$ and $P n$ refer, respectively, to global and partial synchronization in the single-neuron attractor with $n$ spikes per train. 

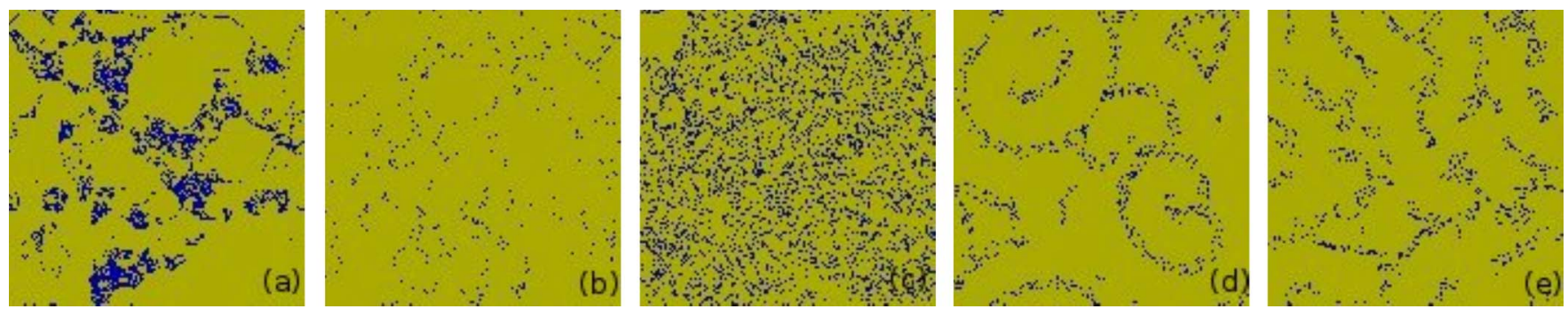

FIG. 8. (Color online) Snapshots of the network activity in some regions of phase diagram: (a) global synchronization with two spikes per train $(G 2), I=1.35, \epsilon=0.025$; (b) partial synchronization with two spikes per train $(P 2), I=1.35, \epsilon=0.045$; (c) and (d), respectively, global and partial synchronization, $I=1.37, \epsilon=0.17(G+P)$; (e) partial synchronization, $I=1.355, \epsilon=0.17(Q+P)$.

there is only quiescence, independently of the initial conditions. In $Q+P$, quiescence and partial synchronization coexist, with random initial conditions ending in a quiescent state $Q$. In $P$, random initial conditions are driven to a partial synchronization state. In $G+P$ and $Q+P$, a partial synchronization state can be attained by starting in $P$ and then slowly varying $I$ or $\epsilon$. This is the way the uniform distribution of Fig. 4 above was generated.

To summarize, single-neuron-type or collective-type dynamics can be found, respectively, at the left or at the right of the dot-dashed line. The lower part of this line is defined by the lower limit of partial synchronization for decreasing $I$, as shown in Fig. 5. This line extends to high $\epsilon$ and low $I$. For example, it passes by the points $(\epsilon, I)=(0.2,1.256),(0.3$, $1.092)$ and $(0.4,0.788)$ (not shown in the figure).

As an illustration of the network behavior in different phases, some snapshots are shown in Fig. 8. It is worth discussing each of them. In (a), there is an example of global synchronization at low- $\epsilon$ regime (G2). This picture corresponds to the period of maximal activity, and half a period after (or before) the membrane potential of all neurons are at the rest value. In (b), there is an example of partial synchronization at low- $\epsilon$ regime $(P 2)$. Here, the network activity is constant in time. The two pictures (c) and (d) correspond both to $I=1.37$ and $\epsilon=0.17(G+P)$. In (c), we have the period of maximal activity of global synchronization $(G)$. At the minimal activity period (not shown) all the neurons are at rest. In (d) one can observe pattern formation (double spirals) with constant activity. It is metastable since, for this set of parameters, random initial conditions drive the network to global synchronization. A dynamic state like (d) is stable in region $P$ of Fig. 7. Finally, (e) is also a metastable regime with constant activity and pattern formation corresponding to $I=1.355$ and $\epsilon=0.17(Q+P)$. Quiescence is the stable state with this set of parameters.

The above results should be viewed in the context of cooperative behavior between the interactive neurons. It is known that the isolated HR neuron is periodic for $I \lesssim 3.28$ [6]. The largest network Lyapunov exponent for $P$ and $G$ attractors is clearly positive, and this was confirmed for a network of different sizes and transient times up to 200000 t.u. Concerning these facts, we can draw the following conclusions: (i) the collective behavior produces a chaotic collective evolution, although the corresponding evolution of an isolated neuron is periodic; (ii) the hysteresis shows that, deep inside the region where the isolated neuron is quiescent, the collective behavior keeps the network active and chaotic. This can be explained similarly to what happens with the quartic map [16]. The isolated neuron has both a stable fixed point (the quiescent attractor) with a large basin of attraction and an unstable fixed point with a small window mapping the unstable to the stable one. When the interaction is turned on, and the whole network is into the basin of attraction of the chaotic attractor, the collective behavior prevents it from finding its way back to the quiescent attractor.

\section{CONCLUSIONS}

In the present work a 2D network of HR neurons was investigated in the vicinity of the activity transition, where the isolated neuron passes from a quiescent to a periodic, active state. Each neuron is uniformly coupled through electrical bonds to all neighbors closer than the interaction range $R$. We have shown that, depending on the coupling strength, there are two different kinds of local attractors, and that this determines the whole network behavior. At low coupling there is a single-neuron-type local attractor, in which the neuron activity is sensitive to the current $I$. Depending on the initial conditions the network globally or partially synchronizes and, in the last case, pattern formation and traveling waves can be observed. If $\epsilon \geq 0.15$ there is the coupledneuron attractor, which is less sensitive to the external current and, indeed, supports the network activity at very low $I$ values.

Partial synchronization, that allows for pattern formation, is relevant from a biological point of view. In this simple model of uniform electrical couplings it can be attained at appropriate initial conditions in a wide region in the $\epsilon-I$ plane. In a narrow region at large $\epsilon$ it can be attained with random initial conditions. It is a matter for further investigation the role that nonuniform electrical coupling, as well as more complex synaptic connections, can play.

\section{ACKNOWLEDGMENTS}

This work was supported in part by the Brazilian agencies CNPq (Conselho Nacional de Desenvolvimento Científico e Tecnológico), FAPERGS (Fundação de Amparo à Pesquisa do Estado do Rio Grande do Sul), and CAPES (Coordenação de Aperfeiçoamento de Pessoal de Nível Superior). The authors want to thank the referees, whose careful criticism helped to improve the quality of the manuscript. 
[1] J. L. Hindmarsh and R. M. Rose, Proc. R. Soc. London, Ser. B 221, 87 (1984).

[2] R. D. Pinto, P. Varona, A. R. Volkovskii, A. Szücs, H. D. I. Abarbanel, and M. I. Rabinovich, Phys. Rev. E 62, 2644 (2000).

[3] A. Szücs, P. Varona, R. Volkovskii, H. D. I. Abarbanel, M. I. Rabinovich, and A. I. Selverston, J. Comput. Neurosci. 11, 1 (2000).

[4] R. Erichsen, M. S. Mainieri, and L. G. Brunnet, Phys. Rev. E 74, 061906 (2006).

[5] R. Huerta, M. Bhazenov, and M. I. Rabinovich, Europhys. Lett. 43, 719 (1998).

[6] M. S. Mainieri, R. Erichsen, Jr., and L. G. Brunnet, Physica A 354, 663 (2005).

[7] M. I. Rabinovich, R. D. Pinto, H. D. I. Abarbanel, E. Tummer, G. Stiesberg, R. Huerta, and A. Selverston, Network Comput.
Neural Syst. 13, 487 (2002).

[8] T. L. Ribeiro and M. Copelli, Phys. Rev. E 77, 051911 (2008).

[9] R. FitzHugh, Biophys. J. 1, 445 (1961).

[10] J. Nagumo, S. Arimoto, and S. Yoshizawa, Proc. IRE 50, 2061 (1962).

[11] J. L. Hindmarsh and R. M. Rose, Nature (London) 296, 162 (1982).

[12] P. Manneville, Dissipative Structures and Weak Turbulence (Academic, New York, 1990).

[13] L. Brunnet and H. Chaté, Physica A 257, 347 (1998).

[14] G. Benettin, L. Galgani, A. Giorgilli, and J.-M. Strelcyn, Meccanica 15, 21 (1980).

[15] E. Ott, Chaos in Dynamical Systems (Cambridge University Press, Cambridge, England, 1993), Chap. 4, p. 136.

[16] L. C. Martins and L. G. Brunnet, Physica A 296, 119 (2001). 